\title{
R-matrices for the semicyclic representations of $U_{q} \widehat{s l}(2)$
}

\author{
I.T.Ivanov* and D.B.Uglov \\ Department of Physics, State University of New York at Stony Brook \\ Stony Brook, NY 11794-3800
}

9 March 1992

\begin{abstract}
R-matrices for the semicyclic representations of $U_{q} \widehat{s l}(2)$ are found as a specialization in the R-matrices of the checkerboard chiral Potts model
\end{abstract}

The chiral Potts model [1] [2] is the first example of a new class of integrable statistical mechanical models whose Boltzmann weights are meromorphic functions on an algebraic variety of genus greater than 1 . From the mathematical point of view this model is related to the theory of cyclic representations of the quantum algebra $U_{q} \widehat{s l}(2)$ at $q^{N}=1[3]$. This relation is expressed by the fact that the R-matrix intertwining two tensor products of cyclic representations is built up of the Boltzmann weights of the chiral Potts model [3] [-

In this paper we consider certain limit of the chiral Potts model which in the algebraic language corresponds to the descent from the cyclic representation to the semicyclic one [6]. Boltzmann weights remain well defined in this limit and as a result we obtain R-matrices which intertwine semicyclic representations of $U_{q} \widehat{s l}(2)$ and satisfy the Yang-Baxter equation.

Series of R-matrices were obtained for semicyclic representations of $U_{q} \operatorname{sl}(2)$ in [7] [8]. The R-matrices obtained here for the larger algebra $U_{q} \widehat{s l}(2)$ may be specialized to $U_{q} s l(2)$ subalgebra. We checked that in the case $N=3$ the Rmatrix from [7] is equivalent by a similarity transform to the R-matrix obtained as a specialization in the chiral Potts model in this letter.

\footnotetext{
${ }^{0}$ e-mail iti@dirac.physics.sunysb.edu
} 
1. Checkerboard chiral Potts R-matrix The chiral Potts model Boltzmann weights

$$
\frac{W_{\xi, \eta}(i)}{W_{\xi, \eta}(i-1)}=\frac{\mu_{\xi}}{\mu_{\eta}} \frac{y_{\eta}-x_{\xi} \omega^{i}}{y_{\xi}-x_{\eta} \omega^{i}}, \quad \frac{\bar{W}_{\xi, \eta}(i)}{\bar{W}_{\xi, \eta}(i-1)}=\mu_{\xi} \mu_{\eta} \frac{x_{\xi} \omega-x_{\eta} \omega^{i}}{y_{\eta}-y_{\xi} \omega^{i}}
$$

depend on two points $\xi$ and $\eta$ of two identical algebraic curves $C_{\xi}$ and $C_{\eta}$ defined by the relations

$$
\mu^{N}=\frac{k^{\prime}}{1-k x^{N}}=\frac{1-k y^{N}}{k^{\prime}}
$$

and the same for $C_{\eta}$. Also $k^{2}+k^{2}=1$.

We shall associate [4] with every cyclic representation $\pi_{\xi}$ of the quantum algebra $U_{q} \widehat{s l}(2)$ two copies of the curve (2) which we shall denote by $C_{\xi}$ and $C_{\xi^{\prime}}$. The variables of the sets $\xi=x, y, \mu$ and $\xi^{\prime}=x^{\prime}, y^{\prime}, \mu^{\prime}$ as well as $k$ are functions of the parameters of the representation. Then the R-matrix intertwining tensor products of two cyclic representations $\pi_{\xi}$ and $\pi_{\eta}$ is given by [⿰]𠃌

$$
\begin{array}{r}
R(\xi, \eta)=\sum_{i, j, k, l=1}^{N} \omega^{i(j-k)}\left(Z^{j} \otimes Z^{k}\right)\left(X^{-1} \otimes X\right)^{i+l} \times \\
\bar{W}_{\xi, \eta}(k) \bar{W}_{\xi^{\prime}, \eta^{\prime}}(j) \widehat{W}_{\xi^{\prime}, \eta}(i) \widehat{W}_{\xi, \eta^{\prime}}(l)
\end{array}
$$

where $Z$ and $X$ are $N$ by $N$ matrices satisfying :

$$
Z X=\omega X Z \quad Z^{N}=X^{N}=1 \quad \omega=q^{2}
$$

and $\widehat{W}_{\xi, \eta}$ is a Fourier transform of the Boltzmann weight $(1): \widehat{W}_{\xi, \eta}(i)=$ $\sum_{k=1}^{N} W_{\xi, \eta}(k) \omega^{-i k}$.

$$
\frac{\widehat{W}_{\xi, \eta}(i)}{\widehat{W}_{\xi, \eta}(i-1)}=\frac{\mu_{\eta} y_{\xi}-\mu_{\xi} y_{\eta} \omega^{i-1}}{\mu_{\eta} x_{\eta}-\mu_{\xi} x_{\xi} \omega^{i}}
$$

Throughout this paper we consider only the case $\mathrm{N}$ odd .

Matrix elements of the R-matrix (3) are vertex weights of the checkerboard chiral Potts model [2], [5]

2. Taking limit in the Boltzmann weights. Here we take a special limit in the checkerboard chiral Potts model which will allow us to get the R-matrices for the semicyclic representations. It is shown in the appendix A that taking 
the semicyclic limit in the cyclic representation is equivalent to the following rescaling of the variables :

$$
\begin{array}{r}
k=\varepsilon^{-N} K, \quad k^{\prime}=\varepsilon^{-N} K^{\prime} \\
x=\varepsilon X, \quad y=\varepsilon^{-1} Y, \quad \mu=\varepsilon^{-1} M, \quad x^{\prime}=\varepsilon^{-1} X^{\prime}, \quad y^{\prime}=\varepsilon Y^{\prime}, \quad \mu^{\prime}=\varepsilon M^{\prime}
\end{array}
$$

and subsequently setting $\varepsilon$ to zero. The Boltzmann weights entering the Rmatrix are invariant under this rescaling :

$$
\begin{aligned}
& \frac{W_{\xi^{\prime}, \eta}(i)}{W_{\xi^{\prime}, \eta}(i-1)}=\frac{M_{\xi^{\prime}}}{M_{\eta}} \frac{Y_{\eta}-X_{\xi^{\prime}} \omega^{i}}{Y_{\xi^{\prime}}-X_{\eta} \omega^{i}} \quad \frac{W_{\xi, \eta^{\prime}}(i)}{W_{\xi, \eta^{\prime}}(i-1)}=\frac{M_{\xi}}{M_{\eta^{\prime}}} \frac{Y_{\eta^{\prime}}-X_{\xi} \omega^{i}}{Y_{\xi}-X_{\eta^{\prime}} \omega^{i}} \\
& \frac{\bar{W}_{\xi, \eta}(i)}{\bar{W}_{\xi, \eta}(i-1)}=M_{\xi} M_{\eta} \frac{X_{\xi} \omega-X_{\eta} \omega^{i}}{Y_{\eta}-Y_{\xi} \omega^{i}} \quad \frac{\bar{W}_{\xi^{\prime}, \eta^{\prime}}(i)}{\bar{W}_{\xi^{\prime}, \eta^{\prime}}(i-1)}=M_{\xi^{\prime}} M_{\eta^{\prime}} \frac{X_{\xi^{\prime}} \omega-X_{\eta^{\prime}} \omega^{i}}{Y_{\eta^{\prime}}-Y_{\xi^{\prime}} \omega^{i}}
\end{aligned}
$$

The finite parts of the parameters $X, Y \ldots$ obey the following equations :

$$
M^{N}=\frac{k^{\prime}}{1-K X^{N}}=\frac{\varepsilon^{2 N}-K Y^{N}}{K^{\prime}} \quad M^{\prime N}=\frac{k^{\prime}}{\varepsilon^{2 N}-K X^{\prime N}}=\frac{1-K Y^{\prime N}}{K^{\prime}}
$$

with $K^{2}+{K^{\prime}}^{2}=\varepsilon^{2 N}$. Let us now take the limit $\varepsilon \rightarrow 0$. The Boltzmann weights (7-8) remain well defined. The equations of the curve show that $M$ is proportional to $Y$ and $M^{\prime}$ is prpoprional to $1 / X^{\prime}$. So the Boltzmann weights $(8,9)$ simplify and to describe them more neatly we introduce one more set of parameters $A, B, C, D$ defined by

$$
A=K^{1 / N} Y^{-1} \quad B=-K^{1 / N} X \quad C=K^{1 / N} X^{-1} \quad D=-K^{1 / N} Y^{\prime}
$$

This set of parameters satisfy the equations of two Fermat curves :

$$
A^{N}=B^{N}+1 \quad C^{N}=D^{N}+1
$$

The Boltzmann weights entering the R-matrix finally have the following form :

$$
\begin{gathered}
\frac{W_{\xi, \eta^{\prime}}(i)}{W_{\xi, \eta^{\prime}}(i-1)}=\frac{D_{\eta}-B_{\xi} \omega^{i}}{C_{\eta}-A_{\xi} \omega^{i}} \quad \frac{W_{\xi^{\prime}, \eta}(i)}{W_{\xi^{\prime}, \eta}(i-1)}=\frac{C_{\xi}-A_{\eta} \omega^{i}}{D_{\xi}-B_{\eta} \omega^{i}} \\
\bar{W}_{\xi, \eta}(i) \\
{\overline{W_{\xi, \eta}}}_{\xi-1)}\left(i-\frac{B_{\xi} \omega-B_{\eta} \omega^{i}}{A_{\xi}-A_{\eta} \omega^{i}} \quad \frac{\bar{W}_{\xi^{\prime}, \eta^{\prime}}(i)}{\bar{W}_{\xi^{\prime}, \eta^{\prime}}(i-1)}=\frac{C_{\eta} \omega-C_{\xi} \omega^{i}}{D_{\eta}-D_{\xi} \omega^{i}}\right.
\end{gathered}
$$

The periodicity property $W(i+N)=W(i), \bar{W}(i+N)=\bar{W}(i)$ is guaranteed by the equations (12). 
3. R-matrix for the semicyclic representations of $U_{q} \widehat{s l}(2)$ Here we present the R-matrix and associated Hamiltonian.

The R-matrix intertwining two semicyclic representations $\pi_{\xi}$ and $\pi_{\eta}$ (See Appendix A) as a result of the previous section is also given by the formula (3) with Boltzmann weights $(13,14)$. The intertwining condition can be proved along the same lines as in the periodic case [4]. To prove the Yang-Baxter equation we introduce four auxiliary Boltzmann weights :

$$
\begin{gathered}
\frac{W_{\xi, \eta}(i)}{W_{\xi, \eta}(i-1)}=\frac{1-\phi B_{\xi} A_{\eta} \omega^{i}}{1-\phi A_{\xi} B_{\eta} \omega^{i}} \quad \frac{W_{\xi^{\prime}, \eta^{\prime}}(i)}{W_{\xi^{\prime}, \eta^{\prime}}(i-1)}=\frac{\phi C_{\xi} D_{\eta}-\omega^{i}}{\phi D_{\xi} C_{\eta}-\omega^{i}} \\
\frac{\bar{W}_{\xi^{\prime}, \eta}(i)}{\bar{W}_{\xi^{\prime}, \eta}(i-1)}=\frac{\omega-\phi C_{\xi} B_{\eta} \omega^{i}}{1-\phi D_{\xi} A_{\eta} \omega^{i}} \quad \frac{\bar{W}_{\xi, \eta^{\prime}}(i)}{\bar{W}_{\xi, \eta^{\prime}}(i-1)}=\frac{\phi B_{\xi} C_{\eta} \omega-\omega^{i}}{\phi A_{\xi} D_{\eta}-\omega^{i}}
\end{gathered}
$$

We checked that the Boltzmann weights (13-14),(15-16) satisfy a set of eight star-tringle equations

$$
\sum_{d=1}^{N} \bar{W}_{q, r}(b-d) W_{p, r}(a-d) \bar{W}_{p, q}(d-c)=R_{p, q, r} W_{p, q}(a-b) \bar{W}_{p, r}(b-c) W_{q, r}(a-c)
$$

with $p, q$ and $r$ replaced by both primed and unprimed indices and $\phi$ arbitrary number. This set of equations imply the Yang-Baxter equation for the semicyclic R-matrix of the form (3). The fact that the R-matrix (3) satisfies the Yang-Baxter equation can also be derived from the intertwining property and an indecomposability of the third-order tensor product of the semicyclic representations of $U_{q} \widehat{s l}(2)$ at generic values of the parameters. If the restrictions $D=-q A, B=-q^{-1} C$ are imposed on the parameters $A, B, C, D$ we obtain $e_{0}=f_{1}$, and $f_{0}=e_{1}$ in the representation of $U_{q} \widehat{s l}(2)$ (see Appendix A). And the resulting R-matrix intertwins between the representations of finite dimensional quantized algebra $U_{q} s l(2)$. In the case $N=3$ we checked that this R-matrix is equivalent to the R-matrix of [7] by the similarity transformation.

The spin-chain hamiltonian corresponding to the R-matrix (3) can be obtained using the standard procedure [2]. To write down it we introduce the following operators:

$$
X_{i}=1 \stackrel{1}{1} \otimes \cdots \otimes \stackrel{i}{X} \otimes \cdots \otimes \stackrel{L}{1} \quad Z_{i}=1 \stackrel{1}{1} \otimes \cdots \otimes \stackrel{i}{Z} \otimes \cdots \otimes \stackrel{L}{1}
$$

where $i=1, \cdots, L ; \mathrm{L}$ is the length of the periodic lattice, $\mathrm{X}$ and $\mathrm{Z}$ are the same as in (4).In terms of these operators the hamiltonian reads as follows:

$$
H=\sum_{j=1}^{L} h_{j, j+1}
$$


$h_{j, j+1}=\sum_{n=1}^{N} F(n) X_{j}^{-n} X_{j+1}^{n}+\sum_{n, m=1}^{N}\left[G_{+}(n, m) X_{j}^{-n} X_{j+1}^{n} Z_{j}^{m}+G_{-}(n, m) X_{j}^{-n} X_{j+1}^{n} Z_{j+1}^{m}\right]$

where the explicit form of the coefficients $F, G_{+}, G_{-}$is given in the appendix B. The form of this hamiltonian is the same as of the general checkerboard chiral Potts model. In the case of the well-known chiral Potts model the coefficients $G_{+}(n, m)$ and $G_{-}(n, m)$ become proportional to $\delta_{n, 0}$ and the hamiltonian acquires the usual form [1].

\section{Appendix A}

In this appendix we describe the $U_{q} \widehat{s l}(2)$ algebra and its cyclic and semicyclic representations at $q^{N}=1$. After that we describe in detail the limiting procedure used in section 2 .

Quantized enveloping algebra $U_{q} \widehat{s l}(2)$ is generated by the elements $t_{i}, t_{i}^{-1}, e_{i}$ and $f_{i}(i=0,1)$ subject to the relations

$$
\begin{array}{r}
t_{i} t_{i}^{-1}=t_{i}^{-1} t_{i}=1 \quad t_{i} t_{j}=t_{j} t_{i} \\
t_{i} e_{j} t_{i}^{-1}=q^{a_{i j}} e_{j}, \quad t_{i} f_{j} t_{i}^{-1}=q^{-a_{i j}} f_{j}, \quad\left[e_{i}, f_{j}\right]=\frac{t_{i}-t_{i}^{-1}}{q_{i}-q_{i}^{-1}} \delta_{i j}
\end{array}
$$

with $a_{i j}=2, i=j$ and $a_{i j}=-2, i \neq j$. These generators also obey the Serre relations.

Tensor products of representations are formed using the following comultiplication

$$
\begin{array}{r}
\Delta\left(e_{0}\right)=e_{0} \otimes 1+z^{-1} t_{0} \otimes e_{0}, \quad \Delta\left(e_{1}\right)=e_{1} \otimes 1+z t_{1} \otimes e_{1} \\
\Delta\left(f_{0}\right)=f_{0} \otimes t_{0}^{-1}+z \otimes f_{0}, \quad \Delta\left(f_{1}\right)=f_{1} \otimes t_{1}^{-1}+z^{-1} \otimes f_{1} \\
\Delta\left(t_{i}\right)=t_{i} \otimes t_{i}, \quad \Delta(z)=z \otimes z
\end{array}
$$

$z$ is central element commuting with the algebra $U_{q} \widehat{s l}(2)$. N-dimensional cyclic representation of the quantum algebra $U_{q} \widehat{s l}(2)$ may be described in terms of the two matrices $Z$ and $X$ and five complex parameters $\xi=\left(a, b, x_{0}, x_{1}, c\right)$,

$$
\begin{array}{r}
\pi_{\xi}\left(e_{0}\right)=x_{0} X^{-1} \frac{b^{2} Z^{-1}-1}{q-q^{-1}}, \quad \pi_{\xi}\left(f_{0}\right)=\left(a b x_{0}\right)^{-1} \frac{a^{2} Z-1}{q-q^{-1}} X \\
\pi_{\xi}\left(f_{1}\right)=\left(a b x_{1}\right)^{-1} X^{-1} \frac{b^{2} Z^{-1}-1}{q-q^{-1}}, \quad \pi_{\xi}\left(e_{1}\right)=x_{1} \frac{a^{2} Z-1}{q-q^{-1}} X \\
\pi_{\xi}\left(t_{0}\right)=\frac{b Z^{-1}}{q a}, \quad \pi_{\xi}\left(t_{1}\right)=\frac{q a Z}{b}, \quad \pi_{\xi}(z)=c
\end{array}
$$


R-matrix is an operator which intertwins tensor products of two representations $\pi_{\xi}$ and $\pi_{\eta}$ taken in an alternated order :

$$
R(\xi, \eta)\left(\pi_{\xi} \otimes \pi_{\eta}\right) \Delta(g)=\left(\pi_{\eta} \otimes \pi_{\xi}\right) \Delta(g) R(\xi, \eta), \text { for all } g \in U_{q} \widehat{s l}(2) \text {. }
$$

Necessary and sufficient condititon for an intertwiner between tensor products of two cyclic representations $\pi_{\xi}$ and $\pi_{\eta}$ to exist is that the following four quantities should be the same in both representations.

$$
\begin{gathered}
\gamma_{1}=\frac{x_{1}^{N}\left(a^{2 N}-1\right)}{1-a^{N} b^{-N} c^{N}}, \quad \gamma_{2}=\frac{a^{2 N}-1}{a^{N} b^{N} x_{0}^{N}\left(a^{N} b^{-N}-c^{N}\right)} \\
\gamma_{3}=\frac{b^{2 N}-1}{a^{N} b^{N} x_{1}^{N}\left(a^{-N} b^{N}-c^{-N}\right)}, \quad \gamma_{4}=\frac{x_{0}^{N}\left(b^{2 N}-1\right)}{1-a^{-N} b^{N} c^{-N}}
\end{gathered}
$$

These four invariants are not independent but obey $\gamma_{1} \gamma_{3}=\gamma_{2} \gamma_{4}$. In order to write the R-matrix for the cyclic case in the factorized form a change of parameters $\left\{\gamma_{1}, \gamma_{2}, \gamma_{3}, \gamma_{4}, a, b, c, x_{0}, x_{1}\right\} \mapsto\left\{k, k_{0}, k_{1}, x, y, \mu, x^{\prime}, y^{\prime}, \mu^{\prime}\right\}$ is convenient.

$$
\begin{array}{r}
k^{2}=\frac{-1}{\gamma_{1} \gamma_{3}}, \quad k_{0}^{N}=-k \gamma_{4}, \quad k_{1}^{N}=-k \gamma_{1} \\
x_{0}=k_{o} x^{\prime}, \quad x_{1}=k_{1} y^{\prime}, \quad a^{2}=\frac{x \mu \mu^{\prime}}{y^{\prime}}, \quad b^{2}=\frac{y}{x^{\prime} \mu \mu^{\prime}}, \quad c=a b \frac{y^{\prime}}{q x}
\end{array}
$$

Then the R-matrix satisfying (29) is given by (3) with the chiral Potts Boltzmann weights.

We will call N-dimensional representation of $U_{q} \widehat{s l}(2)$ semicyclic if $e_{1}^{N}$ and $f_{0}^{N}$ are represented by non-zero scalars while $e_{0}^{N}=f_{1}^{N}=0$. In terms of the parameters of the cyclic representation (26-28) this means $b^{2 N}-1=0$.

Let $\pi_{\xi} \xi=\left(a, b, c, x_{0}, x_{1}\right)$ and $\pi_{\eta} \eta=\left(\hat{a}, \hat{b}, \hat{c}, \hat{x}_{0}, \hat{x}_{1}\right)$ are two cyclic representations. Let also

$$
b^{2 N}-1=\varepsilon^{2 N} f \quad \hat{b}^{2 N}-1=\varepsilon^{2 N} \hat{f}
$$

where $f$ and $\hat{f}$ are some finite functions of the parameters of the corresponding representations and $\varepsilon$ is a number. In limit the $\varepsilon \rightarrow 0$ both representations $\pi_{\xi}$ and $\pi_{\eta}$ become semicyclic. Let us using the equations (32-33) to explicitely single out powers of $\varepsilon$ from the invariants and representation parameters :

$$
\begin{array}{r}
\gamma_{3}=\varepsilon^{2 N} \Gamma_{3}=\varepsilon^{2 N} f G_{3}, \quad \gamma_{4}=\varepsilon^{2 N} \Gamma_{4}=\varepsilon^{2 N} f G_{4} \quad \gamma_{1}=\Gamma_{1}, \quad \gamma_{2}=\Gamma_{2} \\
k=\varepsilon^{-N} K, \quad k_{0}=\varepsilon K_{0}, \quad k_{1}=\varepsilon^{-1} K_{1} \\
x=\varepsilon X, \quad y=\varepsilon^{-1} Y, \quad \mu=\varepsilon^{-1} M, \quad x^{\prime}=\varepsilon^{-1} X^{\prime}, \quad y^{\prime}=\varepsilon Y^{\prime}, \quad \mu^{\prime}=\varepsilon M^{\prime}
\end{array}
$$


As is mentioned in the main text the Boltzmann weights are invariant under this rescaling and we may safely set $\varepsilon$ to zero. The invariants $\Gamma_{1}$ and $\Gamma_{2}$ still play the role of necessary conditions for existing of the intertwiner. The initial invariant conditions for $\gamma_{3}$ and $\gamma_{4}$ become an defining equation for the functions $f$ and $\hat{f}$ from (34):

$$
\frac{f}{\hat{f}}=\frac{\hat{G}_{3}}{G_{3}}=\frac{\hat{G}_{4}}{G_{4}}
$$

The Boltzmann weights entering R-matrix (3) do not depend on the particular choice of $f$ and $\hat{f}$ satisfying (38). The four other possible Boltzmann weights become trivial in the semicyclic limit :

$$
\frac{W_{\xi, \eta}(i)}{W_{\xi, \eta}(i-1)}=1 \quad \frac{W_{\xi^{\prime}, \eta^{\prime}}(i)}{W_{\xi^{\prime}, \eta^{\prime}}(i-1)}=1 \quad \frac{\bar{W}_{\xi^{\prime}, \eta}(i)}{\bar{W}_{\xi^{\prime}, \eta}(i-1)}=\omega \quad \frac{\bar{W}_{\xi, \eta^{\prime}}(i)}{\bar{W}_{\xi, \eta^{\prime}}(i-1)}=1
$$

Appendix B

Here we list the coefficients $G_{+}, G_{-}, F$ entering the hamiltonian (20):

$$
\begin{gathered}
F(n)=\sum_{i=1}^{N} \widehat{W}_{\xi, \xi^{\prime}}(n-i) \widehat{W}_{\xi^{\prime}, \xi}(i)\left[\sum_{s=1}^{n-i} \frac{\gamma-\delta \omega^{s-1}}{C_{\xi}-D_{\xi} \omega^{s}}-\sum_{s=1}^{i} \frac{\beta-\alpha \omega^{s-1}}{B_{\xi}-A_{\xi} \omega^{s}}\right] \\
G_{+}(n, m)=E(n, m) \bar{W}_{\xi^{\prime}, \xi^{\prime}}(m) ; \quad G_{-}(n, m)=E(n,-m) \bar{W}_{\xi, \xi}(m)
\end{gathered}
$$

where

$$
E(n, m)=\sum_{i=1}^{N} \omega^{i m} \widehat{W}_{\xi, \xi^{\prime}}(i) \widehat{W}_{\xi^{\prime}, \xi}(n-i)
$$

and

$$
\begin{array}{r}
\bar{W}_{\xi^{\prime}, \xi^{\prime}}(i)=\frac{\gamma}{C_{\xi}} \frac{\left(\frac{C_{\xi}}{D_{\xi}}\right)^{i}}{1-\omega^{i}} ; \bar{W}_{\xi, \xi}(i)=\frac{\beta}{B_{\xi}} \frac{\left(\frac{B_{\xi}}{A_{\xi}}\right)^{i}}{1-\omega^{-i}} \\
\widehat{W}_{\xi^{\prime}, \xi}(i)=\prod_{s=1}^{i} \frac{C_{\xi}-D_{\xi} \omega^{s-1}}{A_{\xi}-B_{\xi} \omega^{s}} ; \widehat{W}_{\xi, \xi^{\prime}}(i)=\prod_{s=1}^{i} \frac{D_{\xi}-C_{\xi} \omega^{s-1}}{B_{\xi}-A_{\xi} \omega^{s}}
\end{array}
$$

where the parameters $A_{\xi}, B_{\xi}, C_{\xi}, D_{\xi}, \alpha, \beta, \gamma, \delta$ satisfy the following equations:

$$
\begin{aligned}
A_{\xi}^{N} & =B_{\xi}^{N}+1 ; \quad C_{\xi}^{N}=D_{\xi}^{N}+1 ; \\
\alpha & =\left(\frac{B_{\xi}}{A_{\xi}}\right)^{N-1} \beta ; \gamma=\left(\frac{D_{\xi}}{C_{\xi}}\right)^{N-1} \delta ;
\end{aligned}
$$


Summary. Quantum enveloping algebras at roots of unity have hierarchy of irreducible multiparameter representations : cyclic $\rightarrow$ semicyclic $\rightarrow$ nilpotent representations. In this letter we have shown that the process of specializing the parameters in the cyclic representation of $U_{q} \widehat{s l}(2)$ to make it semicyclic goes through on the R-matrix level also. We believe that the R-matrices from [8] are equivalent to the R-matrices obtained here when the later are specialized to the $U_{q} \operatorname{sl}(2)$ case. The limit to the nilpotent representations [10] is under investigation.

The R-matrices for the cyclic representations of $U_{q} \widehat{s l}(2)$ are already known [9] and it seems feasible to find the semicyclic limit in this case.

Acknowdgement

We are grateful to prof. B.M.McCoy for many helpful suggestions and to R.Kedem for discussions.

\section{References}

[1] H.Au-Yang, B.M.McCoy, J.H.H.Perk, S.Tang and M.Yan, Phys. Lett. A 123 (1987) 219; B.M.McCoy, J.H.H.Perk, S.Tang and C.H.Sah, Phys.Lett. A $125(1987) 9$

[2] R.J.Baxter, J.H.H.Perk and H.Au-Yang, Phys.Lett. A 128 (1988) 138

[3] V.V.Bazhanov and Y.G.Stroganov, J.Stat.Phys. 59 (1990) 799

[4] E.Date, M.Jimbo, M.Miki and T.Miwa, RIMS preprint 706 (1990)

[5] R.J.Baxter, V.V.Bazhanov and J.H.H.Perk, Int.J.Mod.Phys.B 4 (1990) 803

[6] P.Roche and D.Arnaudon, Lett. Math. Phys. 17 (1989) 295; G.Keller, Lett. Math. Phys. 21 (1991) 273

[7] C.Gomez, M.Ruiz-Altaba and G.Sierra, Phys.Lett.B 265 (1991) 95

[8] C.Gomez and G.Sierra, Nucl.Phys.B (to appear)

[9] E.Date, M.Jimbo, K.Miki and T.Miwa, RIMS preprint 715 (1990)

[10] A. Berkovich, C. Gomez and G. Sierra, preprint 1992 